\title{
Analysis and interpretation of radiometric signals in a liquid-gas bubble flow
}

\author{
Marcin Zych ${ }^{1, *}$, Robert Hanus ${ }^{2}$, Marek Jaszczur ${ }^{3}$, Anna Golijanek-Jędrzejczyk ${ }^{4}$, Dariusz Świsulski ${ }^{4}$ \\ and Gholam H. Roshani ${ }^{5}$ \\ ${ }^{1}$ AGH University of Science and Technology, Faculty of Geology, Geophysics and Environmental Protection, \\ 30-059 Kraków, Poland \\ ${ }^{2}$ Rzeszów University of Technology, Faculty of Electrical and Computer Engineering, 35-959 Rzeszów, Poland \\ ${ }^{3}$ AGH University of Science and Technology, Faculty of Energy and Fuels, 30-059 Kraków, Poland \\ ${ }^{4}$ Gdańsk University of Technology, Faculty of Electrical and Control Engineering, 80-233 Gdańsk, Poland \\ ${ }^{5}$ Kermanshah University of Technology, Electrical Engineering Department, Kermanshah, 6715685420 Iran
}

\begin{abstract}
The article presents the analysis of signals from a radiometric system consisting of two scintillation probes and two gamma radiation sealed sources. Calculations and interpretation were carried out for the bubble flow of the water-air mixture in the horizontal pipeline. The analysis of the obtained signals was done in time and frequency domain. In the frequency domain, a range of usable frequencies was identified, which were associated with changes in gamma-ray intensity recorded in the time domain. The gas phase velocity, void fraction and statistical parameters of the signal were also calculated.
\end{abstract}

\section{Introduction}

Multiphase flows are currently the subject of intense research, due to the broad application in industry. At the same time, liquid-gas flows are very important from heat transfer enhancement point of view. For example, in $[1,2]$ has been described a two phase flow in microchannels formed by heating the liquid in the cooling systems. A similar topic is taken in $[3,4]$, where the authors analyse the two-phase liquid-gas flows as a result of cooling micro-fins. Due to the boiling in the whole volume, structures of dispersed bubbles are formed in this type of flows. In larger systems, as in the flow through a rectangular channel as presented in [5], the bubbles merge into larger clusters. The phenomena of two-phase liquid-gas flow, bubble type flows is so complex that based on the conducted experiments, further approximate mathematical models are being tried. In the work [6] authors provides an overview and assessment of the functionality of various models describing the path and size of bubbles in boiling liquids. In turn, the analyses presented in [7,8] shown the possibility of performing DNS (Direct Numerical Simulation) simulation for turbulent flow of gas bubbles and dispersed flows in the closed channel.

In almost all examined studies, the authors point out the difficulties associated with measurements and a description of bubble type two-phase liquid-gas flows. In this work, the authors present their view on this issue based on the conducted experiments and using the radioisotope absorption method.

\section{Experimental set-up}

The measuring set-up, discussed in detail in [9], consists of a horizontal pipeline $4.5 \mathrm{~m}$ long with $30 \mathrm{~mm}$ internal diameter. The pump flow velocity has been controlled by the inverter and the valve system enables obtaining the flow (liquid -phase) velocity in the range from 0.15 $\mathrm{m} / \mathrm{s}$ to $3.5 \mathrm{~m} / \mathrm{s}$. To obtain a two-phase flow, the air from the compressor is injected into the flow at a distance $2 \mathrm{~m}$ from the measuring section. Depending on the velocity of the liquid phase and the expense of gas injected, the different flow regimes can be obtained: stratified, slug, plug and bubble. The measuring system has been described in detail in $[9,10]$. It consists of a radiometric set (two $\mathrm{NaI}(\mathrm{Tl})$ scintillation probes and two Am-241 gamma-ray sealed sources), an ultrasonic flow-meter and a gas flow controller. The measurement system allows to measure: the flow velocity of liquid phase (water) $v_{L}$ and gas phase (air) $v_{G}$, void fraction $\alpha$, the amount of gas $Q_{G}$ injected into the research installation.

The gas phase velocity is determined based on a cross-correlation analysis of signals obtained from two scintillation probes. More information on crosscorrelation analysis in radiometric measurements of twophase flows can be found in $[10,11]$.

Determining the flow velocity of liquid and gas does not require calibration at the test section. However, in order to determine the void fraction, a priori calibration of the radiometric set is required, which works as a gamma quanta counter [12]. It is worth to notice that the measuring system is operating on a different basis (mode) than the most commonly used radiometric system functioning as a spectrometer [13,14]. 


\section{Experimental data}

For the signal analysis, the experiment was selected, which was classified as a bubble flow (Fig. 1). The measurement time in this experiment was $480 \mathrm{~s}$, and the sampling time was $\Delta t=1 \mathrm{~ms}$. Table 1 presents the basic parameters of the analysed flow.

Table 1. Basic parameters of water and air flow for the analyzed experiment: $v_{L}$ - water velocity, $\mathrm{u}_{\mathrm{B}}\left(v_{L}\right)$ - uncertainty type $\mathrm{B}$ of water velocity, $v_{G}-$ air velocity, $\mathrm{u}_{\mathrm{c}}\left(v_{G}\right)-$ complex uncertainty of air velocity, $\alpha$ - void fraction, $\mathrm{u}_{\mathrm{c}}(\alpha)$ - complex uncertainty of void fraction, $Q_{G}$ - gas expense, $n$ - rotation velocity of pump, $\mathrm{Fr}^{2}$ - Froude number, Re - Reynolds number.

\begin{tabular}{|c|c|c|c|}
\hline$v_{L}(\mathrm{~m} / \mathrm{s})$ & 2.01 & $\mathrm{u}_{\mathrm{B}}\left(v_{L}\right)(\mathrm{m} / \mathrm{s})$ & 0.15 \\
\hline$v_{G}(\mathrm{~m} / \mathrm{s})$ & 1.010 & $\mathrm{u}_{\mathrm{c}}\left(v_{G}\right)(\mathrm{m} / \mathrm{s})$ & 0.011 \\
\hline$\alpha(-)$ & 0.02696 & $\mathrm{u}_{\mathrm{c}}(\alpha)(-)$ & 0.00083 \\
\hline$Q_{G}(1 / \mathrm{min})$ & 1.60 & $n(\mathrm{rpm})$ & 1600 \\
\hline$F r^{2}(-)$ & 6.88 & $\operatorname{Re}(-)$ & $3.0 \cdot 10^{4}$ \\
\hline
\end{tabular}

The square of Froude's number $F r^{2}$ was calculated according to the relation $[9,14,15]$ :

$$
F r^{2}=\frac{\left(v_{L}-v_{G}\right)^{2}}{g \cdot D_{c h}}
$$

whereas the Reynolds number $\mathrm{Re}$ was defined as follows:

$$
R e=\frac{v_{L} \cdot D_{c h} \cdot \rho}{\eta}
$$

where: $\rho$-density of liquid, $\eta$ - dynamic viscosity, $D_{c h}-$ characteristic dimension for liquid phase, which was define for pipeline with inner radius $r$, as [9]:

$$
D_{c h}=r \sqrt{1-\alpha}
$$

In presented experiments, the air was injected to the pipeline test section through a special nozzle generating a dispersed bubble flow. In contrast to the slug flow $[10,15,16]$, in the bubble flow the bubbles do not have a free surface and are very small, in the order of a few $\mathrm{mm}$. In the further section of the pipeline, bubbles forms clusters, as shown in Fig. 1. By registering such a flow with a camera, the length and depth of the gas bubble clusters were determined.

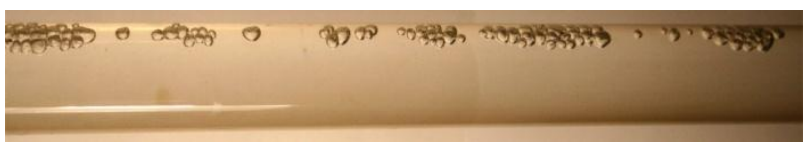

Fig. 1. Flow visualisation made in the analyzed experiment.

Figure 2 shows the definition of the length $l_{k}$ and depth $g_{k}$ of gas bubbles cluster.

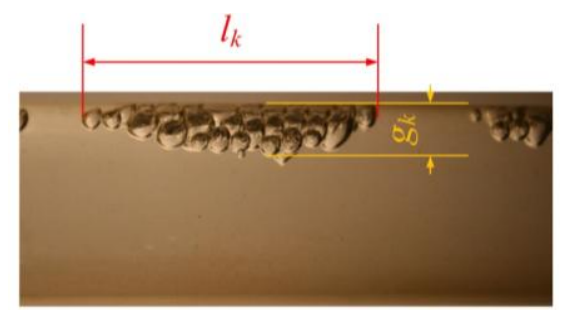

Fig. 2. Presentation of parameters: length $l_{k}$ and depth $g_{k}$ of the gas bubble cluster.

The radiometric measurement system was located where the flow of bubbles was in the form of clusters. Scintillation probes recorded changes in the intensity of gamma radiation. When the bubbles flow between source of radiation and detector the number of registered radiation quanta increased, whereas when no bubbles, the number of counts decreased. The sample signal shows in Fig. 3 illustrates such changes.

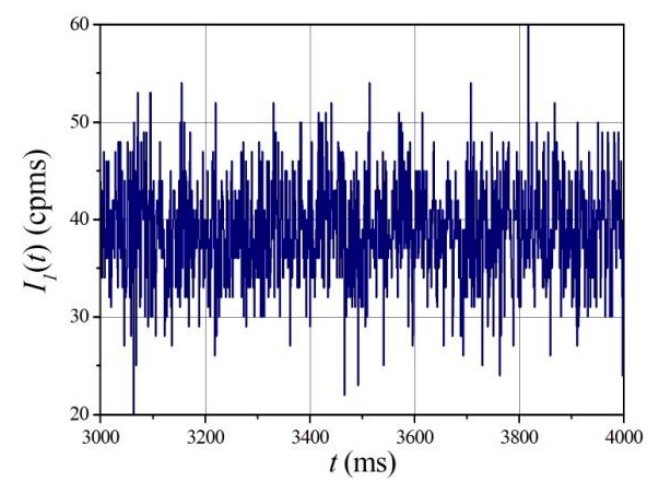

Fig. 3. The part of signal for the analyzed experiment.

The analysis of the signal presented in Fig. 3 is difficult due to a large amount of noise. In such cases, there are many sources of an unfavourable signal-tonoise ratio, among others: the statistical nature of radioactive decay, the chaotic way of forming and the flow of gas bubble clusters. The problem of noise reduction in this type of measurements is discussed in detail in [11].

\section{Signal analysis}

In the frequency domain, cross-spectral density function (CSDF) can be used to analyse signals from scintillation probes. Fig. 4 shows the value of the CSDF module for the signal obtained from analyzed measurements. Due to the significant amount of noise, in order to properly analyse the recorded signal, frequency averaging method was applied (CSDF) [11].

The smoothed value for $|\mathrm{CSDF}|_{\mathrm{S}}$ is shown in Fig. 5. Then a spectral filtering method was applied to obtain a signal without the noise in the time domain [11]. In the $|\mathrm{CSDF}|_{\mathrm{s}}$ plot, the orange frame (dotted line) marked a useful signal registered in range between the frequencies for $0.51 \mathrm{~Hz}$ up to $29.04 \mathrm{~Hz}$. The frequency band outside this range has been removed using a band pass filter. The dominant frequency $f_{0}$, with value is $8.24 \mathrm{~Hz}$, is marked with a continuous red line. 


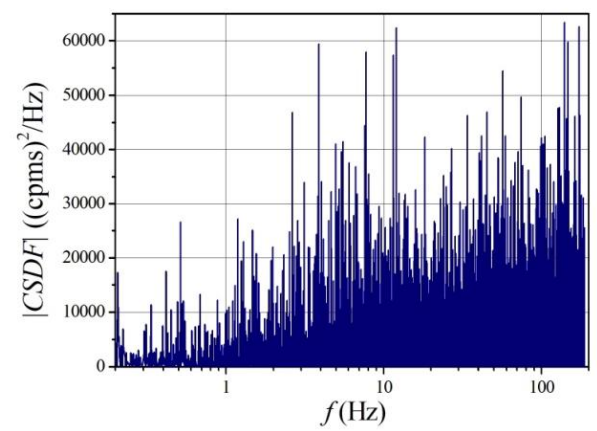

Fig. 4. Module of cross-spectral density function.

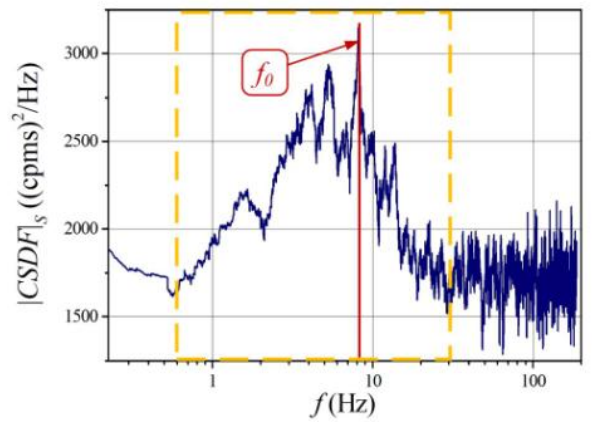

Fig. 5. Smoothed module of cross-spectral density function $|\mathrm{CSDF}|_{\mathrm{s}}$.

After filtering the spectrum, the inverse FFT (Fast Fourier Transformation) was used in order to backward to the time domain. As the result of this procedure is a signal without the influence of low- and high-frequency noise. A part of such a signal is shown in Fig. 6. The negative number of counts $I_{l}{ }^{*}(t)$ are caused by additional signal centring [11] and do not influence signal postprocessing.

Since the signal $I_{l}{ }^{*}(t)$ is related to the size of the cluster bubble flows, this value have been measured from signal presented in Fig. 6 where time duration $t_{k}$ for 50 first peaks is shown. Similarly to analyses presented in [10], $t_{k}$ was related to the cluster's length by the following relationship:

$$
l_{k}=t_{k} \cdot v_{G}
$$

The length uncertainty for the length of clusters measurement was calculated based on the law of propagation of uncertainty [16]:

$$
\mathrm{u}_{\mathrm{c}}\left(l_{k}\right)=\sqrt{\left(v_{G} \cdot \mathrm{u}_{\mathrm{B}}\left(t_{k}\right)\right)^{2}+\left(t_{k} \cdot \mathrm{u}_{\mathrm{c}}\left(v_{G}\right)\right)^{2}}
$$

where: $\mathrm{u}_{\mathrm{B}}\left(t_{k}\right)$ - uncertainty of duration time $t_{k}, \mathrm{u}_{\mathrm{c}}\left(v_{G}\right)-$ combined uncertainty of gas velocity [9-11].

The values of $l_{k}$ for the first 50 clusters of bubbles, together with the average value $\bar{l}_{t}$ (black line), are shown in Fig. 7. The mean value obtained on the basis of time domain analysis is $\bar{l}_{t}=0.064 \mathrm{~m}$, while the uncertainty of measurement $\mathrm{u}_{\mathrm{A}}\left(\bar{l}_{t}\right)=0.006 \mathrm{~m}$. The uncertainty value was calculated based on the dependence:

$$
\mathrm{u}_{\mathrm{A}}\left(\bar{l}_{t}\right)=\frac{\sigma_{l}}{\sqrt{k}}
$$

where: $\sigma_{l}-$ standard deviation of length bubble cluster from analysed probation, $k$ - number of samples (data). Then, the average value of duration time $\bar{t}_{k}=63 \mathrm{~ms}$.

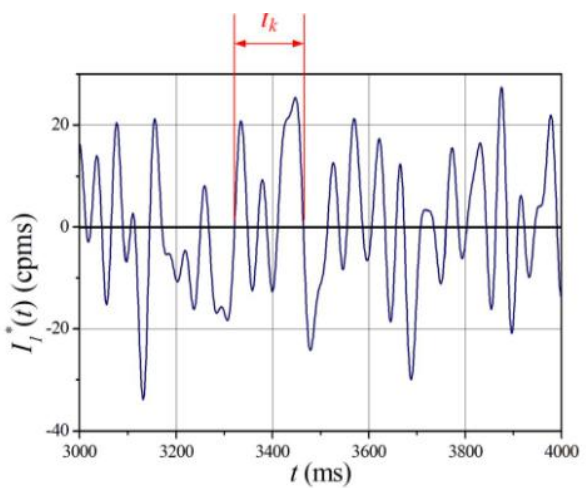

Fig. 6. Short part of filtered signal for analyzed experiment; $t_{k}$. time duration.

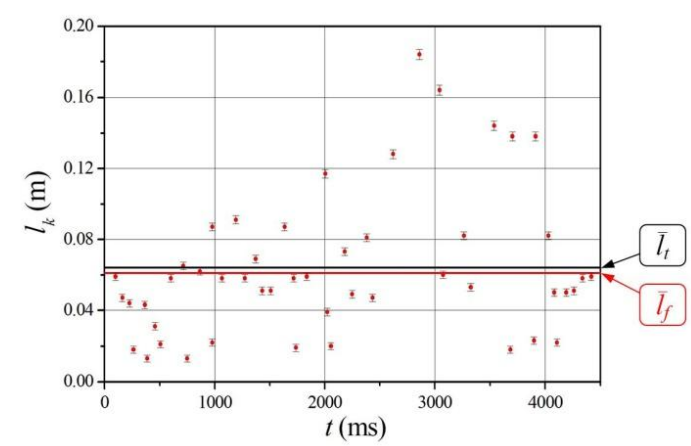

Fig. 7. Distribution of bubbles cluster length $l_{k}$.

During signal analysis is has been seen that the average value of duration time $\bar{t}_{k}$ can be converted into the frequency value [10] as follows:

$$
f_{t}=\frac{1}{\bar{t}_{k}}
$$

For the analysed sample the frequency $f_{t}=15.87 \mathrm{~Hz}$ has been obtained. Comparing this result to the frequency value $f_{0}$, the following relationship can be written:

$$
f_{t} \approx 2 \cdot f_{0}
$$

Hence, the relationship can be derived for the average length of the clusters of gas bubbles, calculated in the frequency domain [10]:

$$
\bar{l}_{f}=\frac{v_{G}}{2 \cdot f_{0}}
$$

In this way, the average value of the bubble cluster length for the entire measurement was obtained $\bar{l}_{f}=$ $0.061 \mathrm{~m}$. In Fig. 7 , the value $\bar{l}_{f}$ is indicated by a solid red line.

Based on the calibration procedure presented in [10], using the Lambert-Beer law [18], it is also possible to calculate the average depths $g_{k}$ of the clusters:

$$
g_{k}=\frac{\ln \left(I / I_{0}\right)}{\mu}
$$


where: $\mu$ - the coefficient of the attenuation of radiation in the mixture, $I$ - the intensity of gamma radiation after the scanning of the mixture, $I_{0}$ - the intensity of radiation registered by the probe for a pipeline filled completely with the water.

In Fig. 8, the distribution of the $g_{k}$ is shown for the analysed experiment. The mean value $\bar{g}_{t}$ is denoted by a solid blue line.

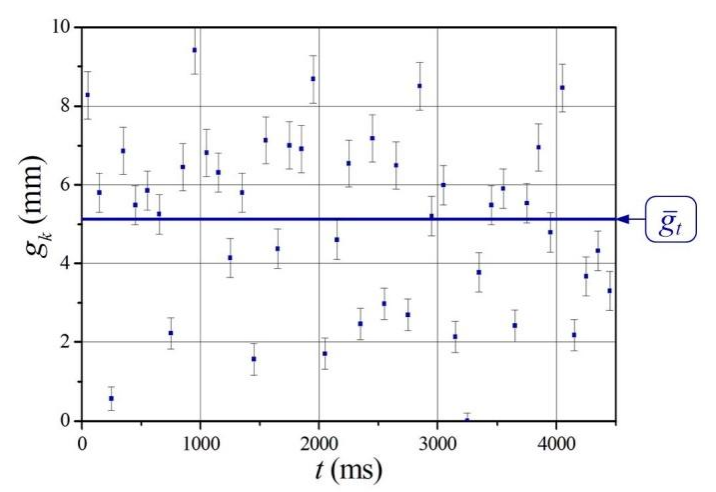

Fig. 8. The distribution of the average values of the depths of bubble clusters $g_{k}$.

The average value of the bubble cluster depth was $\bar{g}_{t}=5.13 \mathrm{~mm}$. The uncertainty of the value $\bar{g}_{t}$ was calculated from the dependence:

$$
\mathrm{u}_{\mathrm{A}}\left(\bar{g}_{t}\right)=\frac{\sigma_{g}}{\sqrt{k}}
$$

where: $\sigma_{g}-$ standard deviation of bubble clusters depth.

The calculated uncertainty equal to $\mathrm{u}_{\mathrm{A}}\left(\bar{g}_{t}\right)=0.29 \mathrm{~mm}$. The results of calculations and images from flow visualisation (Fig. 1, Fig. 2), shows that the obtained values $\bar{l}_{t}, \bar{l}_{f}$ and $\bar{g}_{t}$ are very consistent with the photographic documentation.

\section{Conclusion}

In this work, a sample measurement of the liquid-gas bubble flow made by the radioisotope absorption method was made. It was shown that, despite the high share of noise in the signal, it is possible to obtain precise information about the flow under analysis. In addition to that the evaluation for the flow velocity for both phases (gas and liquid) and for the void fraction, average length and depth values for the gas bubble clusters were enable. The calculations were performed on the basis of signal analysis in time and frequency domain. It was found good agreement of the results for both methods. This was confirmed by the photographic documentation. In addition, the dominant frequency was associated with the physical size of the described flow, which is the length of the gas bubble cluster.

The authors of the article will continue this research studies and, the aim of them will be to generalise the obtained relationships. Presented solution can be seen as a very universal tool, for the system analysis as well as for the wide range of application in the description of other phenomena related to flows, which have been presented, among others, in [19-25].

The authors would like to thank dr Leszek Petryka for his cooperation during the measurements.

This publication is partially financed by AGH University of Science and Technology (Project number 11.11.140.645) and by Polish Ministry of Science and Higher Education under the program "Regional Initiative of Excellence" in $2019-2022$ (Project number 027/RID/2018/19, funding amount 11999900 PLN).

\section{References}

1. K. Strąk, M. Piasecka, B. Maciejewska, Int. J. Heat Mass Transf. 117, 375 (2018)

2. B. Maciejewska, M. Piasecka, Int. J. Heat Mass Transf. 107, 925 (2017)

3. R. Pastuszko, T.M. Wójcik, Exp. Therm. Fluid Sci. 63, 34 (2015)

4. R. Pastuszko, Int. J. Therm. Sci. 125, 197 (2018)

5. T. Ogasawara, H. Takahira, Nucl. Eng. Des. 337, 141 (2018)

6. J. Du, Ch. Zhao, H. Bo, Int. J. Heat Mass Transf. 127, 796 (2018)

7. P. Cifani, J.G.M. Kuerten, B.J. Geurts, Comput. Fluids. 172, 67 (2018)

8. M. Jaszczur, Arch. of Mech. 63, 77 (2011)

9. M. Zych, R. Hanus, M. Jaszczur, V. Mosorov, D. Świsulski, EPJ WoC 180, 02124 (2018)

10. M. Zych, Acta Geophys. (to be published)

11. M. Zych, R. Hanus, B. Wilk, L. Petryka, D. Świsulski, Measurement 129, 288 (2018)

12. M. Zych, R. Hanus, M. Jaszczur, A. Strzępowicz, L. Petryka, W. Mastej, JPCS 745, 032124 (2016)

13. C.M. Salgado, L.E.B. Brandão, C.C. Conti, W.L. Salgado, Appl. Radiat. Isot. 116, 143 (2016)

14. G.H. Roshani, S. Roshani, E. Nazemi, S. Roshani, Measurement 129, 296 (2018)

15. A.O. Morgado, J.M. Miranda, J.D.P. Araújo, J.B.L.M., Int. J. Multiphas. Flow 85, 348 (2016)

16. J.T. García, A. Vigueras-Rodriguez, L.G. Castillo, J.M. Carrillo, Sustainability 9, 402 (2017)

17. Guide to the expression of uncertainly in measurement (International Organization for Standardization 1995)

18. V. Mosorov, Appl. Radiat. Isot. 128, 1 (2017)

19. E. Benachour, B. Draoui, B. Imine, M. Hasnat, L. Rahmani, EPJ Web Conf. 114, 02003 (2016)

20. E. Benachour, B. Draoui, B. Imine, L. Rahmani, B. Mebarki, K. Asnoune, M. Hasnat, Int. Rev. Mech. Eng. 9, 55 (2015)

21. V. Mosorov, Electron. Lett. 42, 630 (2006)

22. V. Mosorov, Part. Part. Syst. Charact. 25, 259 (2008)

23. P. Psota, R. Doleček, V. Lédl, T. Vit, P. Mokrý, P. Dančová, EPJ WoC 143, 02097 (2017)

24. J. Dolanský, Z. Chára, P. Vlasák, B. Kysela, J. Hydrol. Hydromech. 65, 105 (2017)

25. V. Matoušek, Š. Zrostlík, J. Hydrol. Hydromech. 66, 330 (2018) 